\title{
Quality of Service Requirements for the e-learning Grid
}

\author{
Colin Allison \\ School of Computer Science \\ University of St Andrews \\ Scotland \\ ca@dcs.st-andrews.ac.uk
}

\begin{abstract}
In the same way that the Web has evolved from being a technology designed to aid scientific collaboration to one which is employed extensively in e-business and increasingly in e-learning, the Grid is also evolving from its original concept as a highly distributed dynamic source of computing resources "on tap", like the power grid, for e-science, to a means of supporting enterprise computing across heterogeneous, distributed, virtual organisations. However, even the most recent ideas associated with the Grid, as represented by the Open Grid Services Architecture (OGSA) have not yet explicitly addressed e-learning. Considerable experience has been gained over recent years through the development, deployment and management of Distributed Learning Environments (DLEs). This paper draws upon that experience to summarise Quality of Service (QoS) for elearning, and discusses how these can be supported by the OGSA.
\end{abstract}

Keywords: e-learning, GRID, Open Grid Services Architecture, Networks, Quality of Service, QoS, Distributed Learning, Distributed Systems, Web-based Learning

\section{INTRODUCTION}

The proposed exploitation of Grid technologies for e-learning by the LeGE-WG[1] is an example of the growing belief in the power of Distributed Learning Environments (DLEs)[2-4]. Indeed, there is considerable social, economical and political pressure throughout Europe to develop and adopt DLEs in order to meet the goals of wider access, improved quality and reduced costs of education and training. For example, the most recent major government report on the future of higher education in the UK ${ }^{1}$ stated:

"Advances in communications and IT (C\&IT) will radically alter the shape and delivery of
learning throughout the world. Over the next decade ....UK institutions will rely heavily on C\&IT
to teach quality, flexibility and effectiveness of higher education. The potential benefits will
extend to, and affect the practice of, learning and teaching and research. C\&IT will have a
central role in maintaining the quality of higher education in an era when there are likely to be
continuing pressures on costs and a need to respond to an increasing demand for places in
institutions. C\&IT will overcome barriers to higher education, providing improved access and
increased effectiveness, particularly in terms of lifelong learning." [5].

There is a very real danger however that the high expectations being raised by this optimistic belief in the power of C\&IT will result in disillusionment if successful e-learning systems cannot be developed and demonstrated in the near future. Deployment is a major issue, and this is where Grid[6] technologies can help. The form of e-learning that is envisaged by the current

\footnotetext{
1 The Report of the National Committee of Inquiry into Higher Education (1997), chaired by Sir Ron Dearing, was charged with making recommendation s for Higher Education for the next twenty years.
} 
political imperative is premised on the existence of sophisticated distributed systems of exactly the sort that the Grid aims to provide. As there is significant investment being made into creating Grids across Europe it makes sense to obtain added-value from this powerful C\&IT based infrastructure by using it as a deployment vehicle for e-learning.

At this point we note two important points about the recent evolution of the Grid conceptual framework, as represented by OGSA. [7]. Firstly, it is very much a service-oriented infrastructure and the literature has accordingly began to frequently refer to Quality of Service requirements. Secondly, alongside this new emphasis on service-orientation there is a stated belief that Grid technologies are at least as relevant to e-commerce and commerce as they are to e-science. What is clearly missing from this vision is the role of a service-oriented Grid in elearning - especially as education, and e-learning in particular, underpins all future e-science and e-commerce.

The remainder of this paper proceeds as follows: firstly we list pedagogical goals and their technical implications; next we identify the features of DLEs that make them different from more traditional forms of computer-supported learning, and more suitable address the technical requirements of pedagogical goals; we then discuss QoS and Middleware with respect to DLEs, and finally we relate the QoS issues for DLEs to the Open Grid Services Architecture.

\section{PEDAGOGICAL GOALS AND THEIR TECHNICAL IMPLICATIONS}

From the perspective of good educational practice a DLE should provide the following features for students:

- group working should be routinely supported as well as the more traditional model of the solitary learner

- learning resources should be interactive, engaging, and responsive

- real-world input should be easy to incorporate, as should simulations, ranging from simple interactive animations to immersive VR

- students should find themselves at the centre of their online environment, with their individual needs addressed

- wider, more flexible access to educational resources should be provided, often referred to as "anytime/anywhere" learning

Those pedagogical goals imply certain technical requirements.

Support for group working means that online resources may be shared and used by multiple concurrent readers and writers. This requires concurrency control, and raises the usual associated concerns with liveness, safety and fairness. If two or more members of a group are modifying the state of a shared object, at the same time, it is important that the object is not left in an unintended state. If the shared object is distributed, replicated or cached, it is important that different users do not end up with different versions, while believing them to be accurate copies of a single authoritative version. In addition, multi-user awareness is important for teamwork. Members of a group need to know what others are doing, and have appropriate communication options to co-ordinate with each other. For example, text-based, audio or video conferencing may be used to augment shared object interaction. [8]

Interactivity means that online working is much more than simply browsing or downloading lecture notes which have been placed on the Web. Regardless of the balance of computation between server and client the system must be geared to writers as well as readers.

Responsiveness is essential for creating an interactive feel when working across the network. It means that the delay between a user making a request and a result being returned to them should be no longer than some period that is considered reasonable within a particular 
context $^{2}$. If a network service is perceived to be slow then it will be seen as an unproductive use of time, and may be abandoned.

Real world input is facilitated by direct Internet connection to dynamic data sets. For example, the Finesse portfolio management facility [9] allows the management of a portfolio of shares by a student group. The groups maintain their portfolios by buying and selling shares chosen from a database of real-time live values for companies that are quoted on the London Stock Exchange.

Student-centred means understanding users as individuals and implies strong monitoring capabilities, be they for manual inspection, automatic adaptation, or reflective feedback for learners. In an interactive online environment it is of course possible to implement extensive activity logging, which can then be "mined" for useful feedback on a students use of a set of educational resources. This feedback can be used to inform the resource development and adaptation process and thereby enhance the learning environment. The integrity of a student's identity must be carefully guarded in an online setting - their learning credits, prescribed resources and group memberships necessitate appropriate distributed security mechanisms.

Anytime/anywhere implies a resilient, highly available network service. Availability means coping with faults or slow downs in responsiveness. Providing a service based on multiple servers can negate periods of non-availability caused by server crashes and peak loads. These servers may be distributed across a wide area, possibly globally. If the same set of servers is also used to share the load then replication becomes an attractive strategy as it can, in principle, also support performance by providing an appropriate ratio of users to servers. This approach is further useful as a practical means of implementing incremental scalability, which may be necessary to cope with a dynamically changing user population.

\section{DLES VS TRADITIONAL COMPUTER-BASED LEARNING}

DLEs differ in several important respects from earlier Computer Based Learning and Distance Learning scenarios. The ubiquity of the Internet means that any educational institution can now exploit and share its teaching expertise and resources through online access programmes - this type of activity is no longer the preserve of specialist institutions.

Online environments are intrinsically multi-user - where a student may previously have worked in isolation on a computer, DLEs have the potential to i) support teamwork, through various forms of conferencing and shared tasks; ii) introduce real world input into the study environment - for example meteorology students can access satellite weather data in real time and compare their predictions with those of the local news programs.

When a learner is working online it is possible to monitor and analyse the use they make of the system and feed this information back into the educational process, on an individual basis, thereby supporting student-centred learning.

The use of a distributed client-server structure in DLEs means that the QoS being delivered to a user can be monitored and measured. Analysis of this type of information can inform the future development of a DLE's content, architecture and selection of infrastructure options.

DLEs have not only opened up numerous new possibilities for core teaching and learning activities however. In the same way that the application of digital computing has changed the way that scientific programmes are envisaged and carried out, the nature of the entire educational process is also changing. The use of distributed systems in education is affecting not only the content and format of learning resources but also a multiplicity of management tasks such as assignment tracking, marking and grading, student record maintenance and cohort analysis. [10]

\footnotetext{
2 A user working from home over a relatively low bandwidth modem line will have lower expectations than one who is attached to a highspeed campus network.
} 


\section{QUALITY OF SERVICE ISSUES FOR DLES}

Figure 1 illustrates a layered approach to identifying the user-oriented features that a DLE should provide, and the systems services involved in supporting and realising these features. These concerns are mostly found in the middle layer. That is where the pedagogically driven abstractions at the upper layer must be realised using a (largely given) general-purpose lowlevel infrastructure such as the Internet. Note that the middleware terms in bold are referred to frequently in OGSA.

\begin{tabular}{|c|}
\hline Usability Features \\
Abstractions for Tutors and \\
Students Educational Resources, \\
Sessions, Groupwork, Interactivity, \\
Real-world input, User-centric \\
Interfaces, Simulations, Monitoring, \\
Self-Reflection Facilities, \\
Accessibility (W3C) \\
DLE Middleware and QoS \\
Sharing, Security, Concurrency, \\
Availability, Replication, \\
Distribution, Coherence, \\
Responsiveness, Timeliness, \\
Interoperability, Resource \\
Management, Resource Discovery \\
and Location \\
\hline Low-Level Infrastructure \\
Networks, Operating Systems, \\
Server and Client Platforms \\
\hline
\end{tabular}

\section{FIGURE 1: A Layered Overview of Distributed Learning Environments}

The term "Quality of Service" is most closely associated with computer networking, but for QoS to be meaningful to users of a service it should be viewed as a set of systemic properties resulting from the combined behaviour of all the intermediate components between end-users and a service. [11] Support for this holistic view of QoS, can also be found in the ISO Open Distributed Processing program, where it is loosely defined as "... a system or object property, that consists of a set of quality requirements on the collective behaviour of one or more objects"[12].

Middleware in general consists of augmenting a given infrastructure with an extra layer of software that adds value in some useful way, and presenting appropriate programming interfaces and abstractions. With reference to DLEs, the TAGS framework [13] provides support for authentication, access control, group communication and resource management suitable for 
DLEs, that in some way echoes basic OS support for these functions, but at the right level for DLEs. As QoS is often achieved through middleware the final section looks briefly at how key e-learning middleware services and QoS requirements relate to OGSA.

\section{THE RELEVANCE OF THE OPEN GRID SERVICES ARCHITECTURE}

OGSA aims to provide QoS in terms of "common security semantics, distributed workflow and resource management, and application of dynamic ensembles of resources and services (and people)". This relates to aspects of the distributed learning middleware and QoS shown in bold in Figure 1. How can this be exploited in the educational context? Perhaps the best way forward is to look at some scenarios. Before doing so we have to identify the major roles and entities in a distributed learning environment. We discern the roles of learner (student), teacher (lecturer, tutor), resource developer, subject specialist and service provider. So, in a distributed heterogeneous environment a single student may act as the customer of one or more service providers who in turn offer learning resources and tuition services. Any given student may find themselves as a member of several different cohorts (as in a traditional school or university), where these groupings span multiple administrative and organisation domains (unlike a traditional school or university), and where the lifetimes of cohorts and resource allocation vary with the particular learning objectives providing the fundamental focus for their activities.

How can these diverse service and resource providers be integrated into a meaningful usercentric environment for each student? And how can that environment be effectively managed when arbitrary collections of resources have arbitrary longevity? How can the proxy-service approach of OGSA, as opposed to the more traditional model of long-lived cohorts and monolithic resource allocation, support such emerging scenarios? Light may be thrown onto this issue by formulating a detailed mapping between a DLE created within an existing framework for the research, development, deployment and management of Distributed Learning, such as TAGS, and OGSA.

\section{REFERENCES}

1. LeGE-WG, Learning Grid of Excellence - Working Group.

2. Allison, C., Bramley, M., Michaelson, R. and Serrano, J. (1999) An Integrated Framework for Distributed Learning Environments. in "Advances in Concurrent Engineering", 6th ISPE International Conference on Concurrent Engineering. Bath.

3. Allison, C., McKechan, D., Lawson, H. and Ruddle, A. (2000) Quality of Service Issues in Distributed Learning Environments. in Euromicro 2000. Maastricht: IEEE Press.

4. Allison, C., Ruddle, A., McKechan, D., and Michaelson, R. (2001) The Architecture of a Framework for Building Distributed Learning Environments. in Advanced Learning Technologies. Wisconsin: IEEE Press.

5. Dearing, R., Higher Education in the Learning Society" (1997) The Report of the National Committee of Inquiry into Higher Education. HMSO.

6. Foster, I. and Kessleman, C. (1999) The Grid: Blueprint for a New Computing Infrastructure.: Morgan Kaufman.

7. Foster, I., Kessleman, C., Nick, J.M. and Tuecke, S. (2000) The Physiology of the Grid: http://www.globus.org/researh/papers/ogsa.pdf. p. 1-31.

8. Allison, C., Huang, F. and Livesey, M.J. (1999) Object Coherence in Distributed Interaction, in Multimedia'99, N. Correia, T. Chambel, and G. Davenport, Eds. Springer-Verlag: New York. p. $123-132$.

9. Power, D.M., Michaelson, R. and Allison, C (1998). The Finesse Portfolio Management Facility. in 9th CTI-AFM Conference. York: CTI-AFM.

10. Allison, C., Bain, A.L., McKechan, D, and Michaelson, R. (2000) Using TAGS for Distributed IT Project Management. in "The Move from Teaching to Learning", 1st Annual Conefernce of the LTSN Centre for ICS. Edinburgh: LTSN ICS. 
11. Allison, C., McKechan, D. Lawson, H. and Ruddle, A. (2001) An Holistic View of Quality of Service. Interactive Learning Environments. 9(1).

12. ISO/IEC, IS 10476-2. 1996, ISO.

13. Allison, C., McKechan, D., Lawson, H. and Michaelson, R. (2000) The TAGS Framework for Web-Based Learning Environments. in Web Based Learning Environments. Portugal: University of Porto, FEUP Editions. 